Journal of the Society for the Study of Architecture in Canada

Le Journal de la Société pour l'étude de l'architecture au Canada

\title{
Tendances régionales dans la charpente des débuts de l'architecture résidentielle au Québec (1650-1850)
}

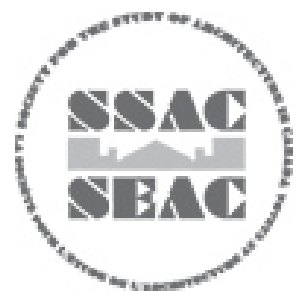

\section{Frédérik Guérin}

Volume 45, numéro 2, 2020

Chercheurs en émergence

Emerging Scholars

URI : https://id.erudit.org/iderudit/1076484ar

DOI : https://doi.org/10.7202/1076484ar

Aller au sommaire du numéro

Éditeur(s)

SSAC-SEAC

ISSN

2563-8696 (numérique)

Découvrir la revue

Citer cet article

Guérin, F. (2020). Tendances régionales dans la charpente des débuts de l'architecture résidentielle au Québec (1650-1850). Journal of the Society for the Study of Architecture in Canada / Le Journal de la Société pour l'étude de l'architecture au Canada, 45(2), 15-26. https://doi.org/10.7202/1076484ar 
FRÉDÉRIK GUÉRIN est candidat au doctorat en histoire de l'art en cotutelle à l'Université du Québec à Montréal et à l'École pratique des hautes études (Paris). Sa thèse porte sur les transformations de l'architecture domestique à Québec entre 1655 et 1755 . Son mémoire de maîtrise, déposé à l'Université Laval (Québec) en 2018, portait quant à lui sur le rôle de la charpente dans l'architecture résidentielle au Québec entre 1650 et 1850. Le présent article en est issu.

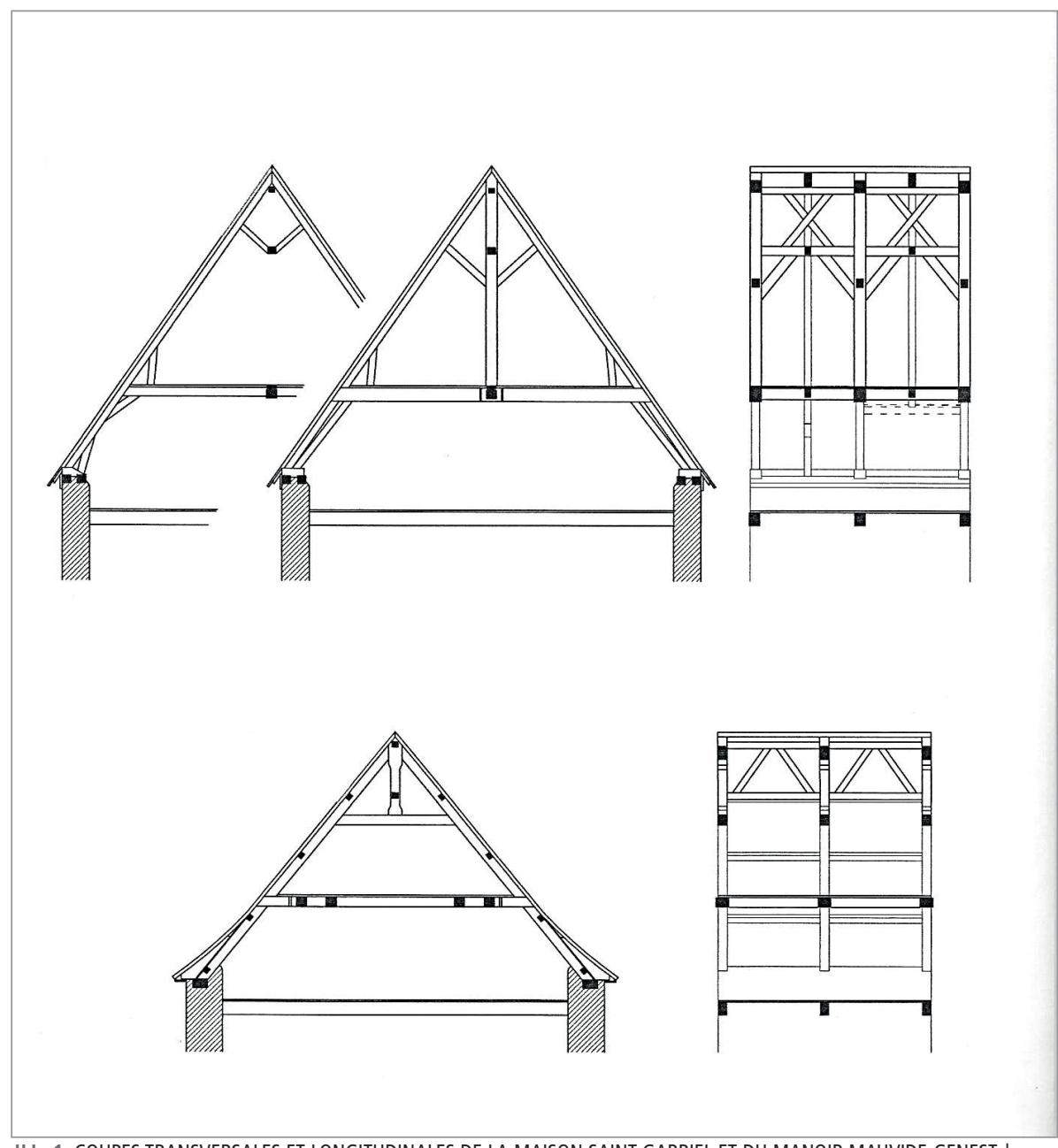

ILL. 1. COUPES TRANSVERSALES ET LONGITUDINALES DE LA MAISON SAINT-GABRIEL ET DU MANOIR MAUVIDE-GENEST.| FRÉDÉRIIK GUÉRIN, 2018.
>FRÉDÉRIK GUÉRIN

$P^{\prime}$ remier apport permanent de l'architecture européenne sur les rives du Saint-Laurent, les maisons d'inspiration française, qui essaiment du dix-septième au dix-neuvième siècle, sont encore aujourd'hui, malgré les ravages du temps, une composante essentielle de l'environnement bâti québécois. Bien que de nos jours la valeur historique de ces maisons soit reconnue et qu'elles soient en bonne partie protégées, elles demeurent encore largement méconnues à plusieurs égards, à commencer par celui de la charpente, héritage d'un savoir-faire dont les premiers développements remontent à plusieurs siècles auparavant en France. Dans cette charpenterie "à la française" ", il s'est établi avec le temps et en réponse à divers problèmes une série d'innovations formelles qui se sont transformées en autant de types pour l'organisation des structures de toitures. Dans l'Hexagone, tout particulièrement dans la partie septentrionale, le comble a acquis pour plusieurs raisons historiques et sociologiques une importance considérable, au point de devenir un élément constitutif et structurant du bâtiment ${ }^{2}$.

Par ailleurs, les recherches sur la charpente préindustrielle française et belge ont considérablement gagné en profondeur et en exactitude depuis le début des années 2000, notamment grâce aux apports de la dendrochronologie ${ }^{3}$ dans le cadre de travaux menés surtout par l'équipe de l'historien de l'art et archéologue belge Patrick Hoffsummer ${ }^{4}$. En se basant sur les formes de représentations traditionnelles de telles structures, ce dernier a également construit une laborieuse, mais efficace méthodologie 


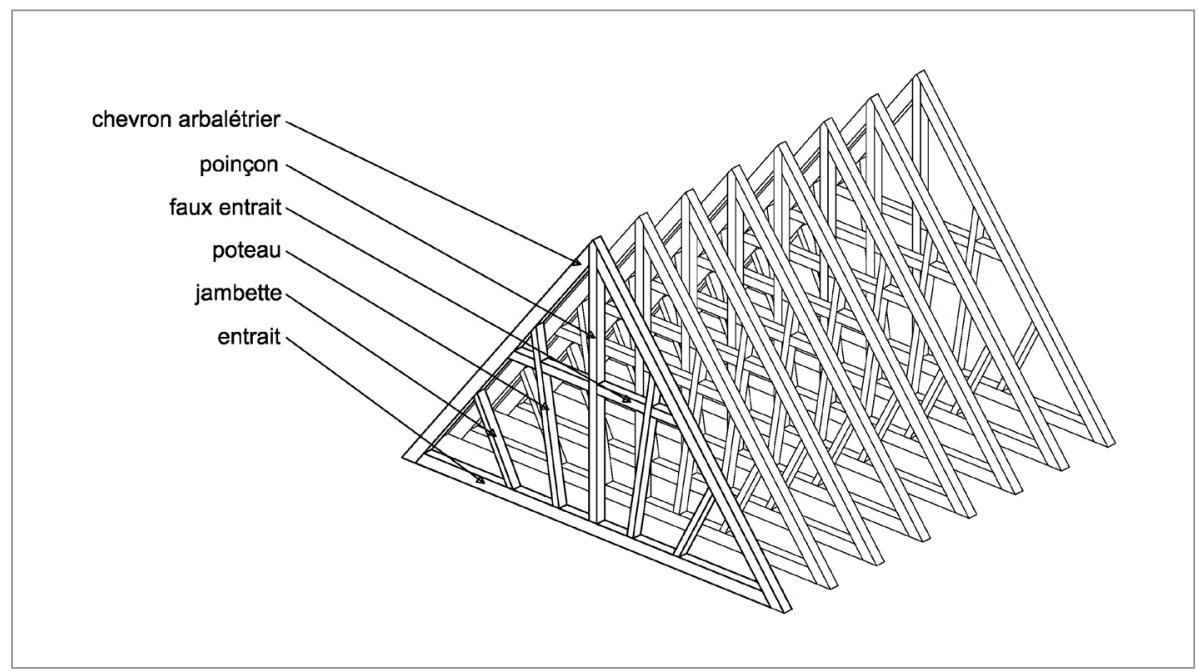

ILL. 2. CHARPENTE À CHEVRONS PORTANT FERMES NON TRAMÉE. | FRÉDÉRIK GUÉRIN, 2020.

de classement et d'analyse typologiques qui comprend pour chaque exemple une coupe transversale et un extrait de la coupe longitudinale ${ }^{5}$. En ajoutant également les travaux de l'archéologue du bâti Frédéric Épaud ${ }^{6}$, on peut maintenant suivre assez précisément tous les développements de la charpente du onzième au dix-neuvième siècle de la moitié nord de la France et de formuler des hypothèses crédibles pour en expliquer les principales transformations.

Arrivant de façon plus soutenue à partir de la seconde moitié du dix-septième siècle, les premiers habitants de la NouvelleFrance ne se sont donc pas présentés les mains vides lorsqu'il s'agissait de monter les charpentes de toitures. Puiser dans un tel répertoire de types de structures et de toits a vraisemblablement permis de répondre aux principaux besoins architecturaux, sociaux et économiques jusqu'à environ cent ans après la fin du Régime français, c'est-à-dire jusqu'au milieu du dix-neuvième siècle. Des recherches réalisées dans le cadre de notre mémoire de maîtrise ${ }^{7}$ ont permis d'analyser ainsi un corpus de plus de quatre-vingt-cinq charpentes réparties le long de la vallée du
Saint-Laurent et mises en place de 1650 à 1850. En nous inspirant de la méthode typologique de Hoffsummer, nous avons dressé des coupes types (ill. 1) pour chacune des variantes désignées de charpente, qui ont permis de faire des comparaisons. Nos observations nous ont permis de constater, sans surprise, I'influence manifeste de la charpenterie des parties nord, est et ouest de la France. Cela était prévisible sachant que la quasi-totalité des colons venus s'établir sur l'actuel territoire québécois provenait de ces régions ${ }^{8}$. Nous avons aussi remarqué un inconstant, mais lent processus de simplification et de standardisation au fil des deux siècles de la période étudiée, les charpentes devenant tranquillement moins complexes et leurs pièces somme toute moins robustes ${ }^{9}$. En fait, la plus grande surprise de notre étude a été la découverte de l'existence, selon toute vraisemblance, de deux «styles» de charpentes durant à peu près toute notre chronologie : celui des maisons de la région de Montréal et celui des maisons de la région de Québec, faute de les avoir nommés autrement. Cela n'a, à notre connaissance, jamais été mentionné par les auteurs qui nous ont précédé sur le sujet.
Précisons d'emblée que ces styles ne sont pas à confondre avec les maisons «normandes " et "bretonnes » que Gérard Morisset voyait à tort dans les deux zones géographiques dans L'architecture en Nouvelle-France $(1949)^{10}$, désignées comme si ces anciennes provinces avaient directement importé leur architecture locale par le biais de leurs habitants, théorie qui n'était pas sans soulever de nombreuses contradictions. L'idée a pourtant perduré pendant quelques décennies avant d'être remise en question par Peter N. Moogk dans Building a House in New France (1977) ${ }^{11}$, puis finalement invalidée par l'historien de l'architecture Luc Noppen à travers divers textes ${ }^{12}$. Ce dernier a néanmoins relevé que si les influences directes bretonne et normande ne tenaient pas la route, il est vrai qu'il a existé en général, au moins pendant un certain temps, une forme de maison globalement distincte dans la région métropolitaine et dans celle de la capitale ${ }^{13}$; nous y reviendrons.

Avant d'aller plus loin sur le sujet, il convient de faire un petit résumé de I'histoire de la charpente traditionnelle française afin de mieux comprendre l'état de la situation au moment de la fondation de la colonie, ainsi que la terminologie que nous allons employer. Commençons par la base : on parle normalement de charpente sous un toit s'il y a présence de "fermes" pour soutenir ses versants, c'est-à-dire de triangles disposés perpendiculairement à l'axe de la cime, le "faîte " $^{14}$. Ce triangle est constitué au minimum d'une pièce de bois horizontale formant la base, appelée "entrait», et de deux pièces obliques disposées sur chaque versant, partant de l'entrait et se joignant au faîte, appelées « arbalétriers » ou «chevrons arbalétriers» (ill. 2) ${ }^{15}$. La ferme peut avoir plusieurs entraits; le premier du bas est défini comme le "grand entrait », ou simplement l'entrait, et les autres sont 


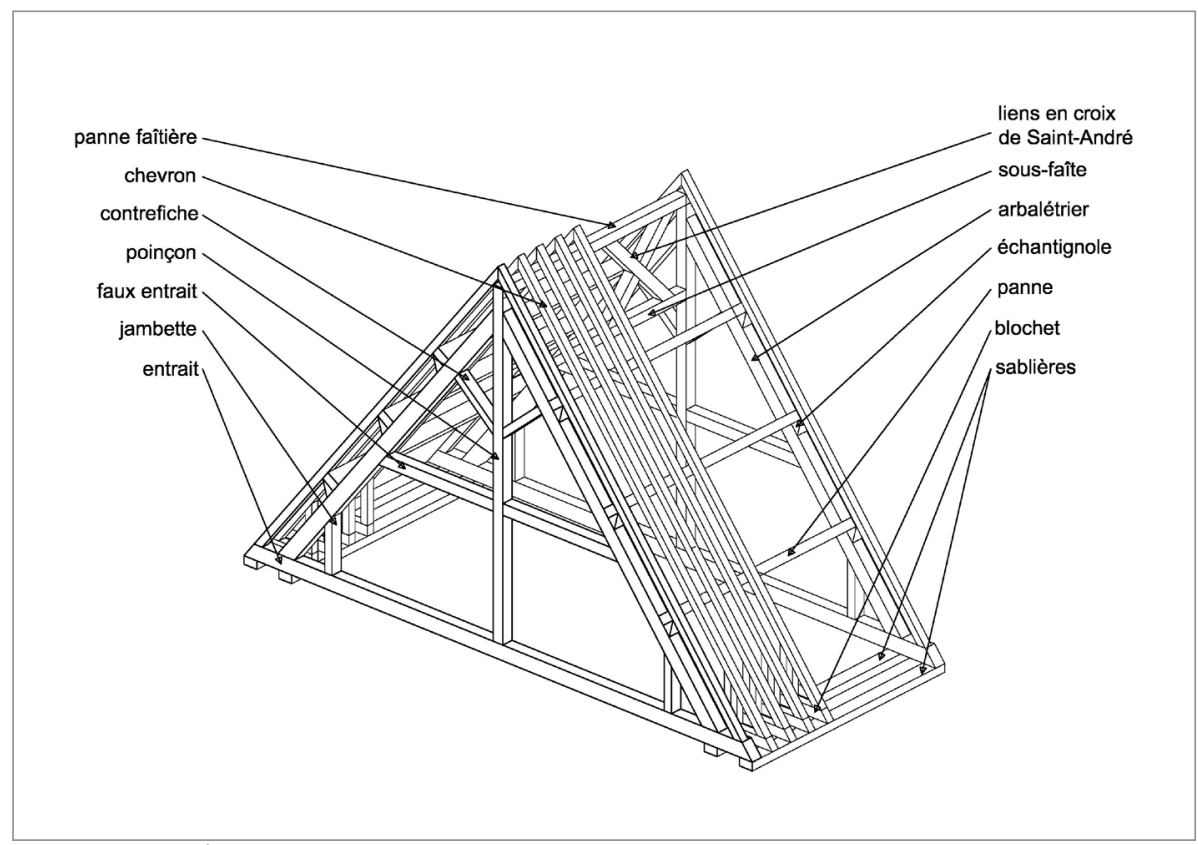

ILL. 3. CHARPENTE À FERMES ET PANNES. | FRÉDÉRIIK GUÉRIN, 2020.

désignés comme des "petits entraits" ou des «faux entraits »" Malgré sa forme triangulaire, une ferme n'est dite «triangulée » que si une pièce verticale appelée «poinçon » part de son faîte et rejoint le milieu de l'entrait, en bas ${ }^{17}$. Dans la charpente préindustrielle que nous étudions, les pièces structurelles sont assemblées sans aucun clou et tiennent par des jonctions à tenons et mortaises, ou à mi-bois, renforcées par des chevilles de bois ${ }^{18}$.

C'est au-delà de ces quelques pièces élémentaires que se trouve la division entre deux grands systèmes historiques regroupant la quasi-totalité des charpentes françaises septentrionales et belges : celui "à fermes et pannes» et celui "à chevrons portant fermes" (ou "à chevrons formant fermes») ${ }^{19}$. Dans le premier, les arbalétriers reçoivent des "pannes", des pièces parallèles à l'axe faîtier sur chaque versant, sur lesquelles reposent les "chevrons", qui sont les pièces obliques plus petites destinées à recevoir la couverture ou les planches (les «voliges») qui la soutiennent (ill. 3) ${ }^{20}$. Les pannes sont maintenues dans la pente des arbalétriers par des petits blocs appelés «échantignolles »"21.

Dans le second système, celui "à chevrons portant fermes", les pannes sont absentes et une seule pièce nommée le «chevron arbalétrier » joue à la fois le rôle du chevron et de l'arbalétrier (ill. 2) 22. Les chevrons arbalétriers, pièces structurales obliques du triangle, reçoivent directement la couverture ou les voliges, d'où le nom. Ce système, commun dans la zone franco-belge, est rare en Europe à l'extérieur de cette région ${ }^{23}$.

Sur ce territoire, les plus vieilles charpentes datées avec certitude par la dendrochronologie remontent au début du onzième siècle ${ }^{24}$. Dans ces charpentes à chevrons portant fermes dites "romanes", les fermes sont toutes semblables (ill. 2), à pente assez faible (entre 30 et 45 degrés) ${ }^{25}$ et souvent sans réel poinçon, ce qui empêche d'atteindre de grandes portées ${ }^{26}$. Pour soutenir le mieux possible la couverture, elles sont alignées de façon serrée sur les murs gouttereaux, une disposition très lourde qui exige une grande quantité de bois. Ces fermes ne possèdent pas de contreventement; elles ne sont donc maintenues en place que par la couverture.

Dans ce qui définit la nouvelle charpente dite "gothique » qui apparaît au douzième siècle dans la portion nord de la France, il y a le passage sans doute débuté dans l'architecture des premières grandes cathédrales, l'opus francigenum, à une forme de charpente où, premier changement majeur, il y a une structure "tramée » où quelques fermes "principales» alternent avec des fermes «secondaires", plus simples (ill. 4) ${ }^{27}$. Cette adaptation suit sans doute la transformation du mur "roman", épais et uniforme pour pouvoir résister à la charge du comble, en mur "gothique», mince et surmonté d'une croisée d'ogives qui répartit la charge par des nervures sur des points précis ${ }^{28}$. Le but est de libérer le centre de chaque travée pour agrandir les ouvertures et maximiser l'apport de lumière naturelle. Le poids doit donc être réduit sur les fermes secondaires qui couvrent le centre de celles-ci et se concentrer, autant que possible, sur les fermes principales qui s'appuient solidement sur les piliers.

Au treizième siècle sont observées les premières charpentes du système à fermes et pannes, qui sont encore plus efficaces pour alléger le centre des travées, puisqu'elles suppriment carrément les fermes secondaires et les remplacent par une série de petits chevrons (ill. 3). Encore mieux, leur poids est presque entièrement redirigé par les pannes sur les arbalétriers des fermes, qui sont dès lors plus espacées et qui permettent des structures ultimement plus légères ${ }^{29}$. 
Deuxième changement majeur et suite logique de la nouvelle dynamique de répartition du poids, la charpente gothique "redresse » les toitures en passant d'une pente qui s'établit autour de quarante-quatre degrés au onzième siècle ${ }^{30}$, puis atteint et se maintient en moyenne autour de soixante degrés du milieu du treizième siècle jusqu'au seizième siècle ${ }^{31}$. Ce phénomène découle d'une demande plus fréquente pour une largeur libre, sans support intermédiaire, qui va au-delà du huit mètres, une situation à peu près inédite à l'époque romane $^{32}$. On rajoute alors de nombreuses pièces de raidissement et de fixation, à commencer par un poinçon au milieu qui permet de trianguler les fermes ${ }^{33}$. Néanmoins, la pression exercée sur les pieds des fermes de ces charpentes toujours plus grandes menace d'écarter les murs vers l'extérieur ${ }^{34}$. C'est à ce moment précis qu'il devient pertinent de redresser les pièces obliques et de rendre la ferme plus verticale et plus pentue : la charge a dès lors moins tendance à pousser sur un plan horizontal et le fait plutôt sur un plan vertical ${ }^{35}$.

Un toit plus haut offre assurément une plus grande prise au vent et, troisième changement majeur, des solutions de contreventements longitudinaux, les «liernes» et les «liens», apparaissent progressivement à partir du début du treizième siècle pour consolider les fermes entre elles autant que pour retransmettre le poids des fermes secondaires vers les fermes principales ${ }^{36}$. On peut sans doute affirmer qu'au début du quatorzième siècle, l'essentiel des techniques et des pièces de la charpenterie traditionnelle française, qui seront en usage pour les cinq cents prochaines années, est maîtrisé, du moins sur les grands chantiers ${ }^{37}$.

Finalement, notons que dans un processus entamé à partir de la fin du quinzième

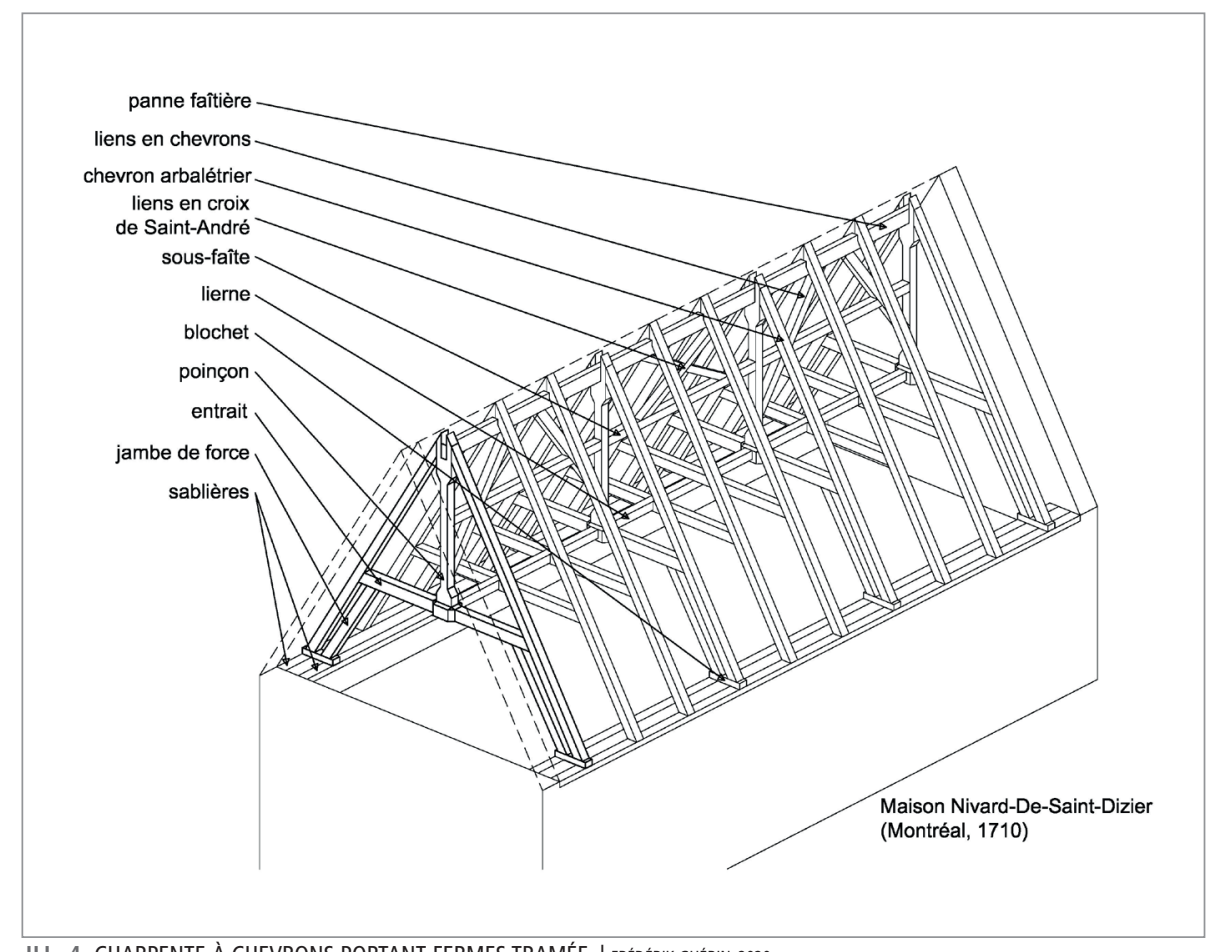

ILL. 4. CHARPENTE À CHEVRONS PORTANT FERMES TRAMÉE. | FRÉdÉRIIK GUÉRIN, 2020.

siècle, l'usage du système à chevrons portant fermes disparaît tranquillement au profit du système à fermes et pannes, qui est plus économique en bois long et massif ${ }^{38}$. La raison est simple : les forêts s'éloignent de plus en plus des grands centres urbains, surtout dans le nord de la France et en Belgique, et la demande de bois de charpente fait face à la concurrence toujours grande du bois de chauffage $^{39}$. Ce changement laisse des traces dans les charpentes de l'époque : les structures s'amincissent, la pente des toitures diminue et l'on observe même au dix-huitième siècle de grands bâtiments à "pannes sans charpente", un système sans ferme avec de simples pannes d'un mur pignon à l'autre, autrefois l'apanage des maisonnettes les plus élémentaires ${ }^{40}$.

Un certaine idée de prestige et de tradition rassurante s'applique probablement aux rares charpentes à chevrons portant fermes construites en France aux dix-septième et dix-huitième siècles comme celle de la chapelle royale du château de Versailles (1705-1710), qui constituent le choix conscient d'une méthode "obsolète", alors même qu'une autre, celle des charpentes à fermes et pannes, est, rappelons-le, plus efficace structurellement, plus légère et plus économique ${ }^{41}$. Épaud suggère dans le même sens que "l'ancienneté, la complexité technique et les moyens importants réclamés par la mise en œuvre - aussi bien en bois qu'en temps - de ce type de structure ont amené les maîtres d'ouvrage à accorder une plus haute estime, empreinte d'un certain respect, aux [chevrons portant fermes] qu'aux fermes et pannes ${ }^{42}$ ". Évidemment, la pénurie de bois et le coût supplémentaire qu'engendre potentiellement le choix de ce système pèsent moins lourd dans les décisions architecturales chez les mieux nantis et dans les régions ayant un accès plus facile à des massifs forestiers comme le Val de Loire et la Bretagne ${ }^{43}$. 


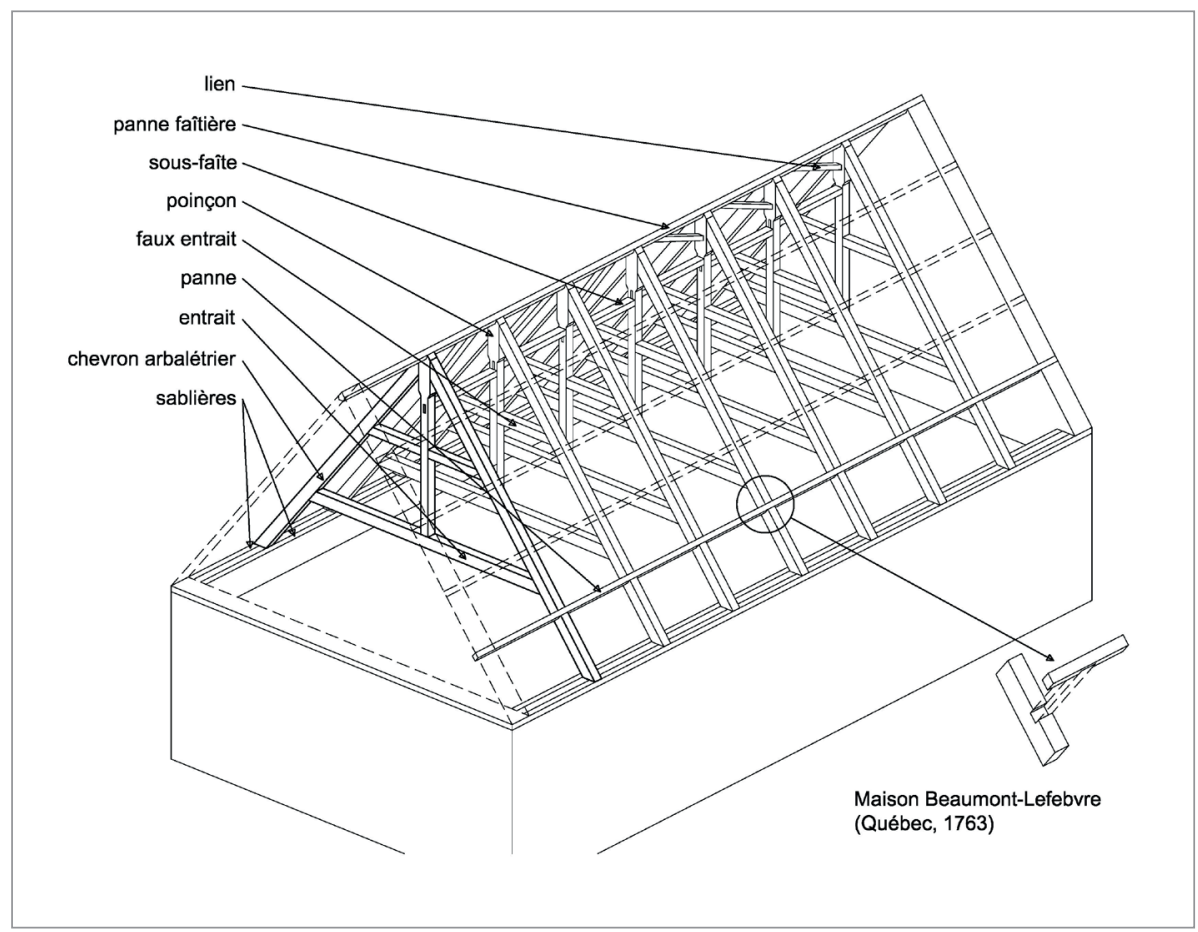

ILL. 5. CHARPENTE À CHEVRONS PORTANT FERMES ET À PANNES EN FILIÈRES. | FRÉdÉRIK GUÉRIN, 2020.

On peut donc conclure sans trop se tromper qu'au moment où les premiers colons et les premiers charpentiers traversent I'océan pour venir s'établir sur les rives du fleuve Saint-Laurent, la charpenterie traditionnelle française est entrée dans sa phase de maturité, essentiellement une transmission d'acquis répétés souvent depuis plusieurs siècles sans changement majeur autre qu'une tendance à la simplification. La situation est donc tout à fait inédite pour ces artisans du dix-septième siècle, habitués à travailler depuis longtemps avec des pénuries de matière première, maintenant dans un "pays neuf» où, c'est bien connu, cette dernière est abondante.

C'est en ayant en tête toutes ces notions que nous avons commencé nos observations et comparaisons typologiques sur les quatre-vingt-cinq charpentes repérées sur soixante-douze bâtiments ${ }^{44}$ au Québec entre 1650 et 1850 . Premier constat : toutes, sauf une ${ }^{45}$, relèvent techniquement du système à chevrons portant fermes, puisqu'elles emploient des chevrons arbalétriers qui font à la fois les fermes et le support de couverture, plutôt que le dédoublement normal du système à pannes entre l'arbalétrier et le chevron pour ces rôles. À lui seul, I'usage manifestement dominant d'un système de charpente qui, rappelons-le, relève déjà de l'«archaïsme » en France au dix-septième siècle, permet d'avancer plusieurs hypothèses intéressantes pour en expliquer la cause. Il faut néanmoins avouer que c'est ici qu'arrive le casse-tête typologique puisque, en réalité, près des deux tiers de ces charpentes à chevrons portant fermes comportent des pannes à la place de fermes secondaires, mais qui sont maintenues par de simples encoches, sans échantignolles (ill. 5).

Il s'agit donc d'une sorte de synthèse qui hybride les deux systèmes et dont nous avons trouvé très peu de traces en France, exception faite d'une certaine parenté avec une famille de charpentes dites "anglo-bretonnes" ou "anglonormandes", qui se retrouvent essentiellement, comme leur nom l'indique, en Bretagne et en Normandie, dans des toitures d'églises, de manoirs et de château $x^{46}$. Ces dernières remontent au moins au quatorzième siècle ${ }^{47}$ et semblent plus abondantes et plus similaires encore à celles du Québec dans l'architecture vernaculaire et rurale, dont nous avons pu trouver quelques exemples, malheureusement impossibles à localiser et à dater précisément. Le tiers restant de notre corpus reste quant à lui plus fidèle aux principes du système à chevrons portant fermes, puisqu'il ne comporte pas de pannes, et dont une partie fait alterner des fermes principales et des fermes secondaires plus légères, alors que l'autre partie fait plutôt alterner de simples «chevrons volants», pièces obliques allant directement du pied au faîte sans autre jonction ${ }^{48}$, mais qui relèvent encore selon nous de l'évocation d'une ferme secondaire. Pour éviter la confusion, nous avons d'ailleurs désigné dans le cadre de notre recherche ces deux grandes familles comme les charpentes "à pannes" pour les structures hybrides et «sans pannes» pour les structures plus classiques.

C'est après avoir dressé ces deux grandes catégories qu'au regard de notre corpus sont apparues deux grandes tendances régionales : celle de l'emploi majoritaire du système à chevrons portant fermes "sans pannes» pour les maisons de la région de Montréal, et celle de l'emploi plus dominant encore du système «à pannes» pour celles de la région de Québec. On peut en voir un exemple avec nos coupes types (ill. 1) de la maison Saint-Gabriel de Montréal (1698) et du manoir Mauvide-Genest de Saint-Jeande-I'île-d'Orléans (1734, agrandi entre 


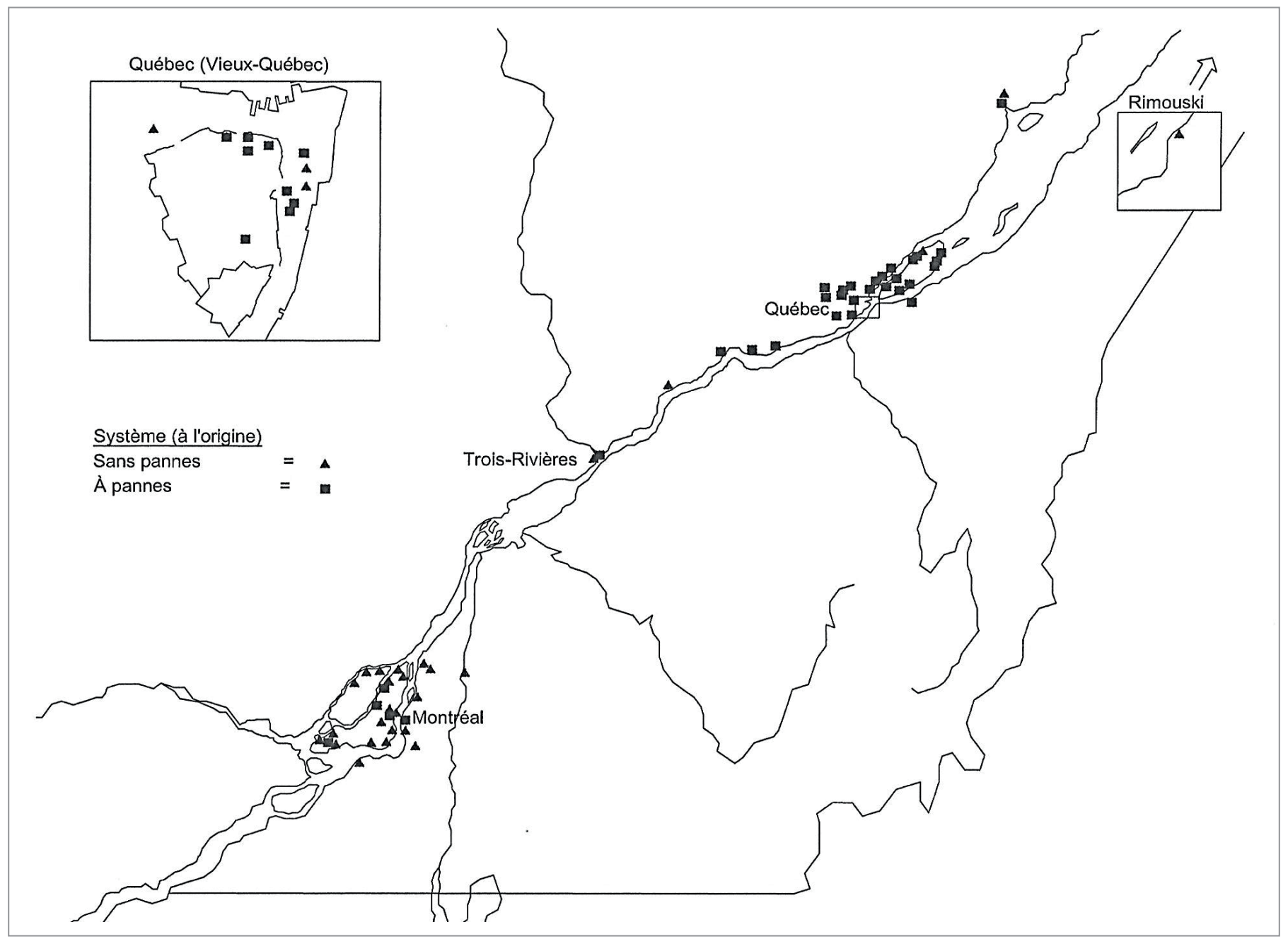

ILL. 6. RÉPARTITION DES CHARPENTES DU CORPUS SELON LES DEUX GRANDS SYSTÈMES (À PANNES ET SANS PANNES). | FRÉdÉRIK GUÉRIN, 2018.

1752 et 1755). D'abord, pour être plus précis, il faut exclure les trois structures à «pannes sans charpente » qui n'entrent dans aucune des deux catégories, ainsi que les trois exemples de la région de Trois-Rivières. Ensuite, sur la carte de la répartition des charpentes du corpus selon les deux grands systèmes (ill. 6), on peut constater leur répartition géographique : quarante-quatre des cinquante et une charpentes à pannes sont dans la capitale et ses environs, alors que vingtdeux des vingt-huit sans pannes sont dans la métropole et ses environs. Le phénomène n'est pas non plus absolu, puisque sept structures à pannes sont présentes dans la région de Montréal, mais seulement à partir de 1723 (première mention : maison Patenaude, Longueuil, 1723). À I'inverse, six structures sans pannes sont présentes dans la région de Québec, surtout au début de la période étudiée, et le dernier exemple qui ne résulte pas d'un agrandissement date de 1743-1745 (maison Lamontagne, Rimouski, première section).

Tout se passe donc comme si, malgré sa persistance jusqu'au milieu du dix-neuvième siècle (la maison Morissette, de
Saint-Charles-sur-Richelieu, construite vers 1830, I'emploie encore) dans la région de Montréal, l'« archaïque » système sans pannes est présent un peu partout au dix-septième siècle et au début du dix-huitième, à la manière de la complexe charpente de la maison SaintGabriel (ill. 7). Le système à pannes, quant à lui, est déjà présent en parallèle dès la plus vieille maison du corpus (manoir de Charleville, Boischâtel, première partie, vers 1665), mais prend du galon surtout à partir du second quart du dixhuitième siècle. Il en vient à dominer, en ville comme en campagne, la région 
de Québec, voire tout le territoire, au début du dix-neuvième siècle, en excluant peut-être une petite poche de résistance métropolitaine. N'oublions pas non plus qu'autour de la capitale, les données sont légèrement faussées par les nombreuses reconstructions suivant la Conquête ${ }^{49}$, qui ont tendance à uniformiser les typologies et qui remplacent peut-être de vieilles charpentes sans pannes par de nouvelles à pannes dans les années qui suivent le changement de régime.

On sait qu'il y a un certain décalage chronologique dans le développement des deux régions, celle de Montréal et de sa couronne immédiate s'étant ouverte légèrement plus tardivement que Québec et ses environs ${ }^{50}$. Mais justement, n'auraitil pas été plus logique de trouver plus d'exemples de la famille la plus ancienne, celle des charpentes sans pannes, dans la seconde, et de voir un plus grand nombre de charpentes à pannes dans la première?

Selon nous, la première piste d'explication passe par la question de l'empreinte au sol, ce que notre corpus est à même de démontrer. Dotées presque toujours d'un corps de logis double (ou une largeur équivalente) après 1720 , les maisons de la région de Montréal sont plus profondes, mais relativement courtes en façade alors que celles de la région de Québec, presque toujours à corps de logis simple, sont moins profondes, mais plus longues. En conséquence, il semble donc que ce soit la largeur du bâtiment, et donc celle des fermes, qui ait la plus grande incidence sur la complexité de ces dernières, qui voient les pièces se multiplier pour franchir une plus grande portée et reprendre un toit un tant soit peu imposant, faisant fi de leur étroitesse dans l'autre direction. Logiquement, les fermes sont donc, règle générale, plus complexes dans la région montréalaise, même pour les maisons "ordinaires", mais en comportent un moins grand nombre. C'est un peu l'inverse pour la région de Québec, ce qui équilibre un peu la quantité totale de pièces dans les structures des deux zones. Nous croyons que dans cette région, cela s'expliquerait par le fait que l'on maintient longtemps la pratique des corps de logis simples, une façon de concevoir le bâtiment qui est encore la norme au dix-septième siècle, en France comme au Canada ${ }^{51}$. Avec ces corps de logis simples, il est coutume de joindre des agrandissements successifs sur la longueur de part et d'autre à partir de la maison d'établissement initiale, à l'origine elle aussi plus carrée ${ }^{52}$. Aux environs de Montréal, I'architecture résidentielle rurale, en moyenne légèrement plus tardive, est rapidement influencée par celles des maisons urbaines ${ }^{53}$. Les travaux de Pierre-Édouard Latouche ont en outre démontré que ces dernières ont gagné en profondeur et ont progressivement pris une forme carrée entre 1700 et 1730, ce qui est attribuable aux réglementations qui limitent la largeur des lots en façade en ville ${ }^{54}$.

Luc Noppen interprète aussi cette particularité comme la preuve architecturale d'une gestion différente des terres. D'une part, celles de la proche région de la capitale sont rapidement saturées dès la fin du dix-septième siècle, ce qui pousse les familles à agrandir, comme nous l'avons constaté sur dix-sept maisons, souvent pour accueillir d'autres générations sur le même lot et dans le même bâtiment ${ }^{55}$. D'autre part, les terres de la région métropolitaine sont longtemps moins compactes et plus grandes, laissant de la place aux familles pour subdiviser le lot et offrir des constructions neuves à chaque génération ${ }^{56}$. Nous l'avons là aussi constaté sur le terrain : il n'y a qu'un seul cas d'agrandissement dans le corpus pour cette zone, la maison Jean-Baptiste-Jamme-Dit-Carrière (Kirkland, entre 1740 et 1761, agrandie

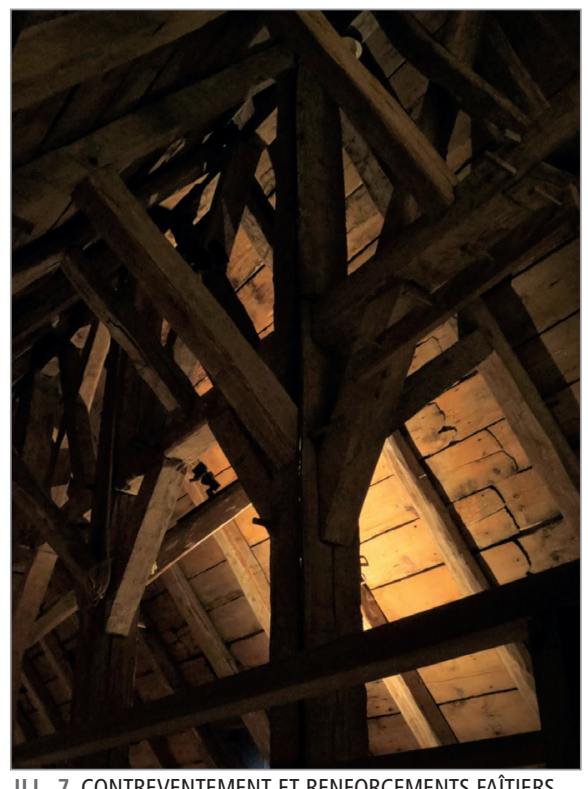

ILL. 7. CONTREVENTEMENT ET RENFORCEMENTS FAITTIERS DE LA MAISON SAINT-GABRIEL. | FRÉDÉRIK GUÉRIN, 2016.

vers 1820); et les autres paraissent être la première maison construite sur le lot ou encore avoir directement remplacé, sans rien conserver, une petite maison d'établissement temporaire. Il serait donc possible d'imaginer que les systèmes «à pannes» et "sans pannes» font tout simplement encore partie du coffre à outils du charpentier de l'époque et que, de façon pragmatique, il s'adapte à la forme du bâtiment sur lequel il va appuyer sa structure.

Autre élément de réponse, qui viendrait surtout expliquer la domination du système à pannes dans la région de Québec : la réglementation en place sous le Régime français concernant la construction des maisons urbaines, dont l'ordonnance de $1721^{57}$ de l'intendant Claude-Thomas Dupuy et celle de $1727^{58}$ de l'intendant Michel Bégon sont probablement les plus importantes. En effet, par crainte des incendies, la première requiert (et la deuxième le répète) d'éviter à tout prix la mise en place de "grosses charpentes», 


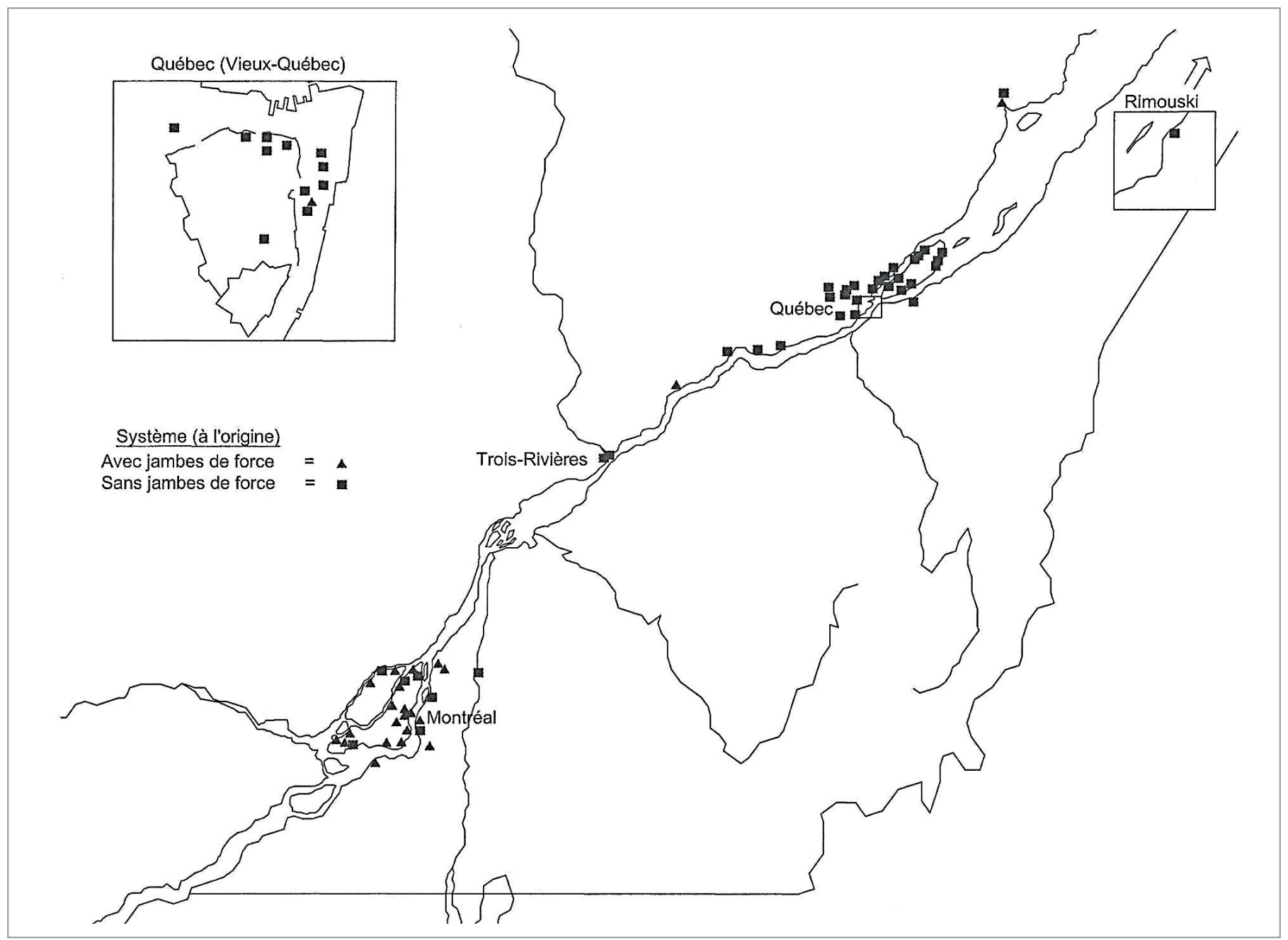

ILL. 8. RÉPARTITION DES CHARPENTES ENTRE CELLES À JAMBES DE FORCE ET CELLES QUI N'EN ONT PAS. | FRÉdéRII GUÉRIN, 2018.

que plusieurs chercheurs ont associées à la version «lourde» des chevrons portant fermes ${ }^{59}$, et de favoriser toutes les structures à "filières", c'est-à-dire à pannes, avec le moins de fermes et de pièces de bois possible ${ }^{60}$. Ces ordonnances, comme beaucoup de lois et règlements du système juridique français prérévolutionnaire, n'ont probablement pas été suivies dans l'immédiat et à la lettre en raison de la résistance des usages - à preuve la réitération ${ }^{61}$-, mais on peut présumer que cela a au moins accéléré une tendance déjà bien entamée en zone urbaine, surtout à Québec. Et comme le siège du pouvoir colonial fait sans doute peser une influence et une surveillance plus grandes dans ses environs immédiats qu'à Montréal, il y a lieu de supposer que les règlements d'urbanisme ont dû déteindre plus facilement dans l'architecture rurale de cette région, ne serait-ce que parce que les charpentiers qui habitent dans la ville travaillent également dans la campagne environnante et qu'ils ont pu y importer les techniques devenues obligatoires.

Pour ajouter à la réflexion, il faut dire que la différence entre les "styles» de charpente montréalais et québécois va au-delà du système de support de la couverture, et se remarque également dans l'usage (ou le non-usage) de certaines pièces. Le plus frappant est sans doute l'emploi de la "jambe de force", cette pièce qui va de l'entrait au plancher ou à la sablière ${ }^{62}$, très fréquente dans la région de Montréal, surtout dans sa forme allant au plancher, mais qui est à peu près absente dans la région de Québec (ill. 8). Notons également que dans cette dernière zone, le poinçon se termine presque toujours à l'entrait du haut s'il y en a deux, alors que dans la zone montréalaise, le poinçon 


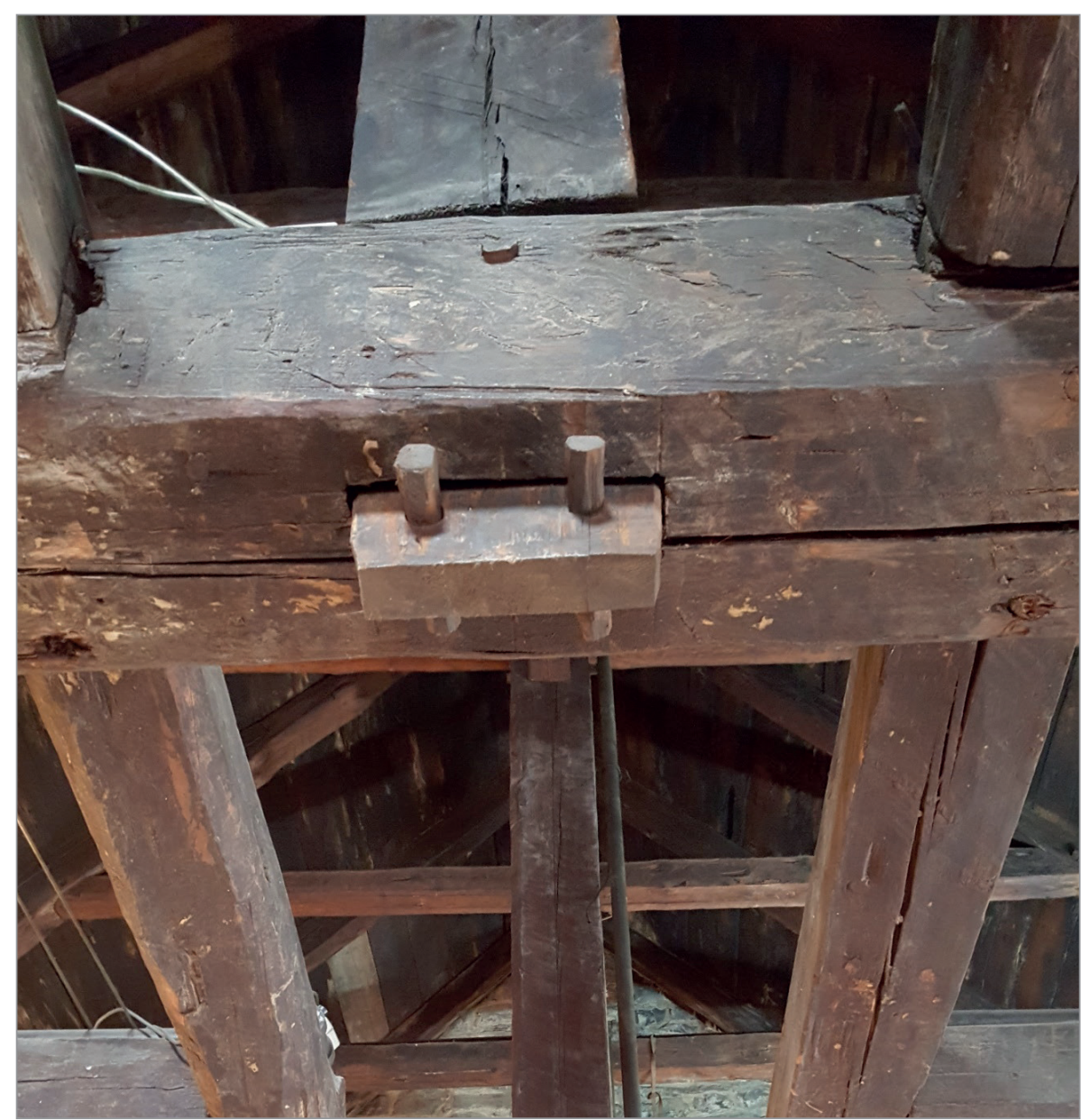

ILL. 9. SUSPENTE DE LA MAISON DU CALVET. | FRÉDÉRRIK GUÉRIN, 2017.

"traverse " près d'une fois sur deux ce poinçon pour aller s'attacher à celui du bas, formant ainsi une sorte de croix. Par ailleurs, il n'y a que là, sur douze charpentes, qu'une "suspente", sorte de clé ronde chevillée, dépasse du poinçon sous l'entrait du bas pour mieux consolider cet ensemble en croix (ill. 9) ${ }^{63}$. Finalement, on peut remarquer que dans les environs de la capitale, la sous-faîtière est presque toujours décalée en hauteur d'une travée à l'autre pour faciliter l'insertion de leurs embouts mortaisés dans le poinçon sans se nuire entre elles (ill. 5). À l'inverse, dans la région métropolitaine, on préfère presque toujours laisser les sous-faîtières au même niveau d'une travée à l'autre et se contenter d'embouts mortaisés moins profondément (ill. 4). Comme quoi la différence entre les deux "styles" de charpente va au-delà de la question de la largeur et du système de support de couvertures. Il serait même possible d'imaginer qu'il ait existé dans les deux régions, pour au moins un temps, deux «écoles de pensée " distinctes de charpentiers, qui se seraient transmis deux façons différentes de voir la charpente, formant des sortes de "modules", particulièrement pour les fermes, adaptables au contexte. Cela reste néanmoins difficile à prouver, faute d'indices suffisants. Seuls les souhaitables développements et raffinements de l'usage de la dendrochronologie ${ }^{64}$ dans le contexte québécois risquent d'apporter quelques utiles précisions pour définir la ligne du temps. Chose certaine, la charpente traditionnelle «à la française » qui subsiste encore au Québec n'a pas fini de révéler tous ses mystères.

\section{NOTES}

1. Nous retenons cette expression en raison de son usage pour désigner la charpenterie traditionnelle française au Québec dans les marchés de construction à partir du début du XIXe siècle face à la " nouvelle " charpenterie de tradition anglaise. Martin, Paul-Louis, 1999, À la façon du temps présent. Trois siècles d'architecture populaire au Québec, Québec, Presses de I'Université Laval, p. 116.

2. Pérouse de Montclos, Jean-Marie, 2013, L'architecture à la française. Du milieu $X V^{e}$ siècle à la fin du XVIII' siècle, Paris, Picard, p. 44.

3. La dendrochronologie désigne le procédé de datation du bois à partir de l'étude des cernes de la croissance des troncs d'arbre. Rey, Alain et Josette Rey-Debove (dir.), 2011 [1967], Le Petit Robert. Dictionnaire alphabétique et analogique de la langue française, Paris, Le Robert, p. 676

4. Voir surtout Hoffsummer, Patrick (dir.), 2002, Les charpentes $d u X I^{\mathrm{e}}$ au XIX $X^{\mathrm{e}}$ siècle. Typologie et évolution en France du Nord et en Belgique, Paris, Centre des monuments nationaux, Monum et Éditions du Patrimoine ; ainsi que, du même auteur, 2011, Les charpentes $d u X I^{e}$ au XIXe siècle. Grand Ouest de la France. Typologie et évolution, analyse de la documentation de la Médiathèque de I'architecture et du patrimoine, Turnhout, Brepols.

5. Hoffsummer, Les charpentes du XI au XIXe siècle. Typologie et évolution en France $d u$ Nord et en Belgique, id., p. 163.

6. Voir surtout Épaud, Frédéric, 2007, De la charpente romane à la charpente gothique en Normandie. Évolution des techniques et des structures aux $X I I^{\mathrm{e}}-X I I^{\mathrm{e}}$ siècles, Caen, Publications du Centre de recherches archéologiques et historiques anciennes et médiévales (CRAHM).

7. Guérin, Frédérik, 2018, Une relecture de la charpente dans I'architecture résidentielle au Québec, 1650-1850, mémoire de maîtrise en histoire de l'art, Université Laval, Québec. Le présent article en est issu. 
8. Trudel, Marcel, 1968, Initiation à la NouvelleFrance. Histoire et institutions, Montréal, Holt, Rinehart et Winston, Limitée, p. 145.

9. II nous faut toutefois avouer que ce constat sur la robustesse repose essentiellement sur des impressions visuelles, le temps ayant manqué dans nos recherches de maîtrise pour faire un relevé systématique des mesures des pièces de chacune des charpentes.

10. Morisset, Gérard, 1949, L'architecture en Nouvelle-France, Québec, Collection Champlain.

11. Moogk, Peter N., 1977, Building a House in New France. An Account of the Perplexities of Client and Craftsmen in Early Canada, Toronto, McClelland and Stewart Limited, p. 22.

12. Voir surtout Noppen, Luc, 1983, "La maison québécoise : un sujet à redécouvrir ", dans Collectif, Questions de culture, $n^{\circ} 4$. Architectures: la culture dans l'espace, Québec, Institut de la recherche sur la culture et Leméac, p. 69-102 ; ainsi que Morisset, Lucie K. et Luc Noppen, 2001, " La maison québécoise : construction et déconstruction d'un emblème ", The Journal of Canadian Art History, vol. 22, n 1, p. 26-67.

13. Noppen, Luc, 1991, "Maison Armand ", dans Commission des Biens culturels du Québec, Les chemins de la mémoire. Monuments et sites historiques du Québec, tome II. Québec, Les Publications du Québec, p. 157.

14. Hoffsummer, Patrick et Alain Prévet, 2002, "Glossaire ", dans Hoffsummer, Les charpentes $d u X I^{e}$ au XIXe siècle. Typologie et évolution en France du Nord, op. cit., p. 356.

15. Id., p. 353.

16. Laframboise, Yves, 1975, L'architecture traditionnelle au Québec. Glossaire illustré de la maison aux $17^{\mathrm{e}}$ et $18^{\mathrm{e}}$ siècles, Montréal, Éditions de l'Homme, p. 133.

17. Épaud, De la charpente romane à la charpente gothique en Normandie, op. cit., p. 609.

18. Hoffsummer et Prévet, "Glossaire ", op. cit., p. 355.

19. Hoffsummer, Patrick, 2002, "Typologie de la charpente ", dans Hoffsummer, Les charpentes $d u X I^{e}$ au $X I X^{e}$ siècle. Typologie et évolution en France du Nord, op. cit., p. 164.

20. Pérouse de Montclos, Jean-Marie, 2003 [1995], Histoire de l'architecture française : de la Renaissance à la Révolution, Paris, Mengès et Éditions du Patrimoine, p. 154.

21. Pérouse de Montclos, Jean-Marie, 2011 [8 éd.] [1972], Architecture. Description et vocabulaire méthodique, Paris, Éditions du patrimoine, p. 150

22. D'après l'expression forgée par Pérouse de Montclos dans Architecture. Description et vocabulaire, op. cit., p. 152

23. Hoffsummer, "Typologie de la charpente ", op. cit., p. 164.

24. Ibid.

25. Hoffsummer, Patrick, 2002, "La forme du toit ", dans Hoffsummer, Les charpentes $d u$ $X I^{\mathrm{e}}$ au $X I X^{\mathrm{e}}$ siècle. Typologie et évolution en France du Nord, op. cit., p. 151.

26. Épaud, De la charpente romane à la charpente gothique en Normandie, op. cit., p. 140.

27. Hoffsummer, Patrick, 2002, « Les faits saillants d'une évolution ", dans Hoffsummer, Les charpentes $d u X I^{e}$ au XIXe siècle. Typologie et évoIution en France du Nord, op. cit., p. 266.

28. Épaud, De la charpente romane à la charpente gothique en Normandie, op. cit., p. 161.

29. Id., p. 215

30. Id., p. 142.

31. Hoffsummer, "La forme du toit ", op. cit., p. 151.

32. Hoffsummer, « Les faits saillants d'une évolution », op. cit., p. 270.

33. Épaud, De la charpente romane à la charpente gothique en Normandie, op. cit., p. 179.

34. Ibid.

35. Hoffsummer, "Typologie de la charpente" op. cit., p. 176.

36. Épaud, De la charpente romane à la charpente gothique en Normandie, op. cit., p. 199.

37. Hoffsummer, « Les faits saillants d'une évolution », op. cit., p. 272.

38. Id., p. 273.

39. Pérouse de Montclos, Histoire de l'architecture française, op. cit., p. 155

40. Hoffsummer, "Les faits saillants d'une évolution », op. cit., p. 277.

41. Pérouse de Montclos, Histoire de l'architecture française, op. cit., p. 154

42. Id., p. 216-217.

43. Hunot, Jean-Yves, 2004, "L'évolution de la charpente de comble en Anjou : XII ${ }_{-}$ XVIII siècles ", Revue archéologique de l'Ouest, vol. 21, n 1, p. 239.

44. Certaines maisons ont deux, voire trois variantes de charpentes différentes au gré des agrandissements. Les dates fournies sont celles estimées pour les charpentes et non pour les bâtiments eux-mêmes, et sont issues des analyses formulées dans notre mémoire de maîtrise.

45. Il s'agit de la charpente de la première section du manoir Boucher-de-Niverville, à TroisRivières (vers 1668), qui appartient clairement au système à fermes et pannes, avec toutefois une forme plus rare en France et en Belgique, dite « à arbalétriers de chambrée ».

46. Hoffsummer, Patrick, Rachel Touzé et Muriel Van Ruymbeke, 2011, "Typologie de la charpente ", dans Hoffsummer, Les charpentes du XI $I^{\mathrm{e}}$ au XIXe siècle. Grand Ouest de la France, op. cit., p. 169

47. Ibid.

48. Laframboise, L'architecture traditionnelle au Québec, op. cit., p. 91-93.

49. C'est le cas pour au moins une dizaine de maisons dans notre corpus, sur une période qui s'échelonne de 1759 à 1768 .

50. Noppen, "La maison québécoise : un sujet à redécouvrir », op. cit., p. 76.

51. Pérouse de Montclos, Histoire de l'architecture française, op. cit., p. 235.

52. Lessard, Michel, 1998, L'île d'Orléans. Aux sources du peuple québécois et de l'Amérique française, Montréal, Éditions de l'Homme, p. 274.

53. Noppen, "La maison québécoise : un sujet à redécouvrir », op. cit., p. 76

54. Latouche, Pierre-Édouard, 2005, Les marchés de construction notariés et les transformations de la sphère privée et de sphère publique à Montréal entre 1700 et 1755 , thèse de doctorat en histoire de I'art, Université Laval, Québec, p. 71-79.

55. Noppen, « Maison Armand », op. cit., p. 157.

56. Ibid..

57. «Ordonnance portant règlement pour la reconstruction des maisons (détruites dans l'incendie du 19 juin 1721, en la ville de Montréal) en matériaux incombustibles, et pour d'autres fins ; du huitième juillet, mil sept cent vingt-un », dans Robert Côté, Françoise Dubé, Mario Savard et Serge SaintPierre, 1992, Portraits du site et de I'habitat de la Place-Royale sous le Régime Français : Annexes, Québec, Les Publications du Québec, p. 106-108.

58. «Ordonnance portant règlement pour la construction des maisons, en matériaux 
incombustibles, dans les villes de la colonie ; du 7 juin, 1727 ", dans Côté et al., id., p. 110-117.

59. Grignon, Marc et Luc Noppen, 1983, L'art de I'architecte. Trois siècles de dessin d'architecture à Québec, Québec, Musée du Québec et Université Laval, p. 120.

60. Côté et al., Portraits du site et de l'habitat de la Place-Royale, op. cit., p. 106 et 112.

61. Ouellet, Marie-Ève, 2018, Le métier d'intendant en France et en Nouvelle-France au XVIII' siècle, Québec, Septentrion, p. 269.

62. Pérouse de Montclos, Architecture. Description et vocabulaire méthodique, op. cit., p. 153.

63. Hoffsummer et Prévet, "Glossaire ", op. cit., p. 358-359.

64. Notons que huit charpentes de notre corpus ont pu être datées avec cette technique. 
de la

Société pour l'étude de

I'architecture au Canada

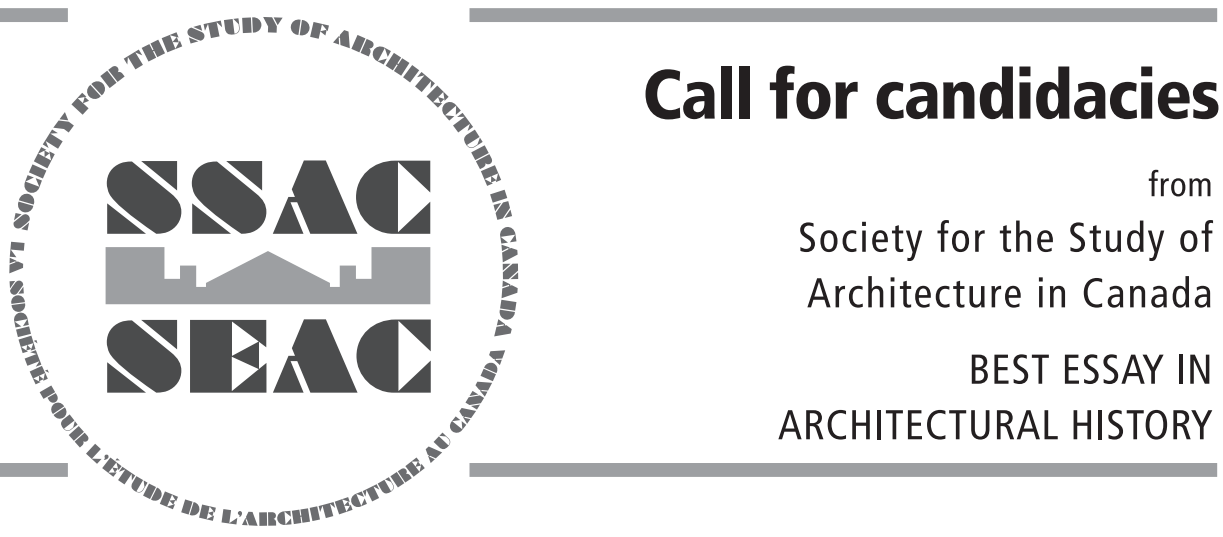

MEILLEUR ESSAI EN

HISTOIRE DE L'ARCHITECTURE

The Martin-Eli-Weil prize is awarded annually by the SSAC to a student who submits an essay on the role played by the built environment in Canadian society. The $\$ 250$ prize and certificate shall be awarded to the winner at the Society's Annual Conference, where he/she will be invited to present a paper on his/her essay. The winning essay shall also be published by the Society in the Journal. All part-time and full-time students registered in a program leading to a university degree in a Canadian university are eligible; submissions from students registered in a foreign university, but dealing with a Canadian subject, are also invited.

The essay, in French or English, may deal with a specific building, contemporary or historic, whether existing or at a proposal stage; a complex of attached buildings; a specific building type (examined for its historical, functional, structural, or aesthetic importance); cultural landscape (parks, cemeteries, farms, etc.); the life of a person who has influenced the built environment; or a philosophical, sociological, or historical issue related to the built environment. Architectural projects that do not strictly comply with the above criteria shall be rejected. Submissions must be presented in hard copy or in electronic format (PDF), double-spaced, and contain from 3000 to 5000 words. The use of visual material is encouraged but their printed format should not exceed $8 \times 10$ inches $(20 \times 25 \mathrm{~cm})$. If applicable, submissions must be accompanied by endnotes and a bibliography, and be supported by conceptual framework in accordance with current standards. Essays will be evaluated according to originality and innovative nature of the subject, as well as accuracy, relevancy of methodology, and writing style.

Essays should be sent to the person in charge of the competition, by February 28, 2021 on paper or in PDF.

By submitting an essay, a candidate accepts the terms of the competition. He/she agrees to present his/her paper at the annual conference and to submit the text to the Journal of the SSAC for publication.

En soumettant son essai au concours, un candidat en accepte les termes. II/elle s'engage à présenter son essai au congrès annuel de la société et à soumettre son texte au Journal de la SEAC pour publication s'il/elle obtient le prix.

\section{Luc Noppen}

noppen.luc@icloud.com

La Société pour l'étude de l'architecture au Canada est une société savante que se consacre à l'examen du rôle que joue l'environnement bâti dans la société canadienne. Parmi ses membres se trouvent des ingénieurs en structure, des architectes de paysage, des historiens de l'architecture, des historiens en urbanisme, puis des urbanistes, des sociologues, des folkloristes et des spécialistes dans des domaines tels la préservation du patrimoine et l'histoire du paysage. Fondée en 1974 sous la direction de l'architecte en conservation Martin Eli Weil, la Société est une association nationale dont l'intérêt se concentre sur l'environnement bâti du Canada sous toutes ses formes.

\author{
Luc Noppen \\ noppen.luc@icloud.com
}

The Society for the Study of Architecture in Canada is a learned society devoted to the examination of the role of the built environment in Canadian society. Among its members are structural engineers, landscape architects, architectural historians, urban historians, urban planners, sociologists, ethnologists, and experts in such fields as heritage conservation and landscape history. Created in 1974 by Martin Eli Weil, a conservation architect, this national Society is mainly interested in all forms of the Canadian built environment. 